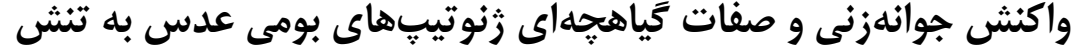

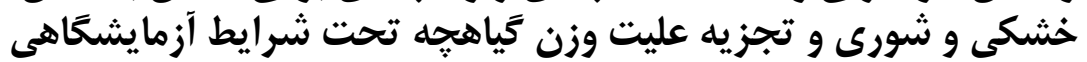

\author{
ورهر ام رشيدى \\ دانشيار كروه زراعت و اصلاح نباتات، واحد تبريز، دانشكاه آزاد اسلامى، تبريز، ايران (نويسنده مسوول: Rashidi.varahram @ gmail.com) \\ تاريخ يذيرش: \\ تاريخ دريافت:
}

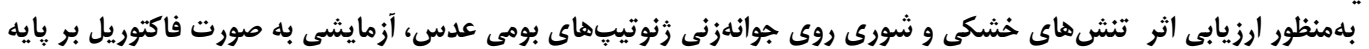

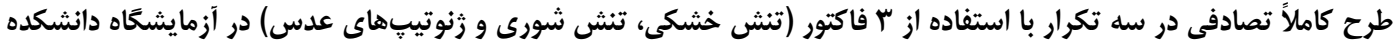

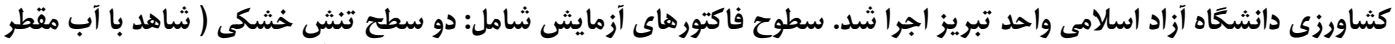

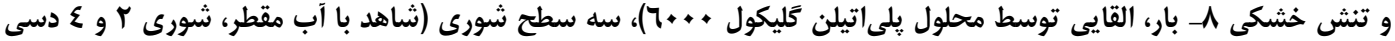

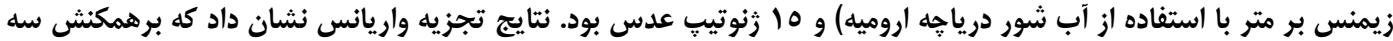

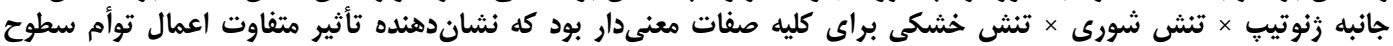

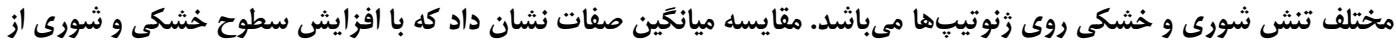

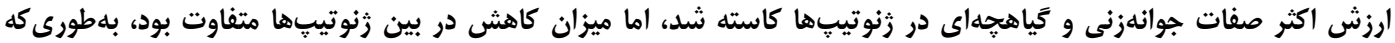

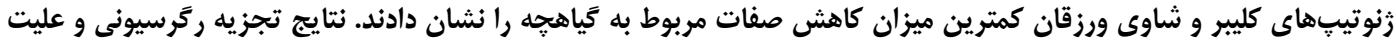

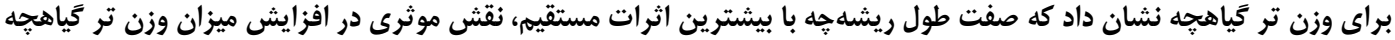
در شرايط تنش شورى و خشكى ديان داشتند.

وازههاى كليدى: تجزيه عليت، خشكى، شورى، عدس، رترسيون

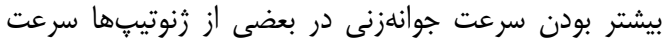

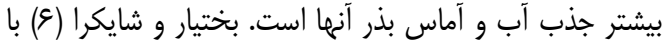

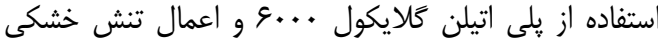

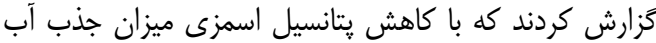

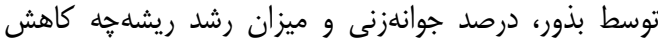

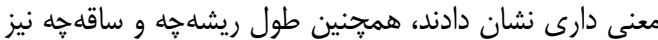

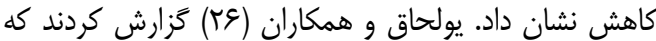

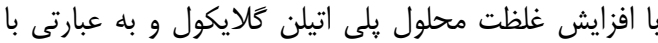

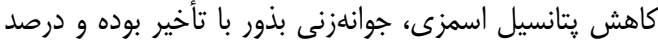

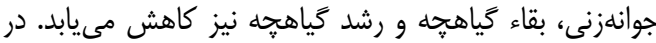

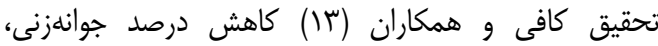

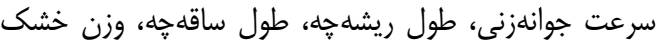

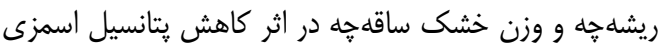

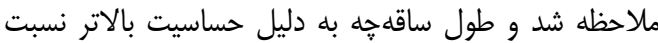

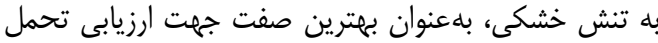

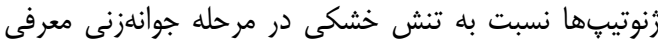

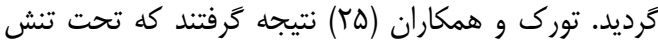

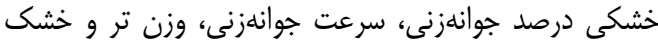

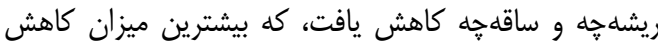

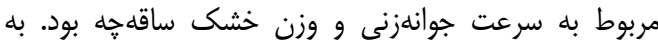

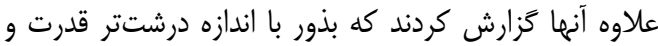

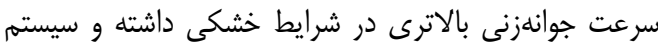

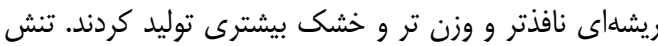

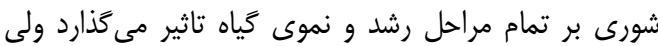

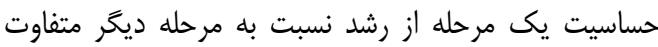

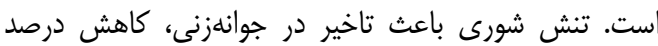

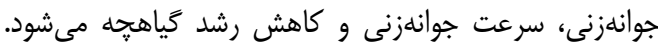
شورى پايين، جوانهزدن را به تاخير مى كىاندازد، اما شورى بالا
عدس (Lens culinaris Madik) به لحاظ استفاده آن

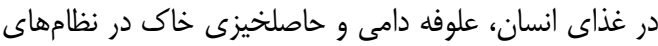

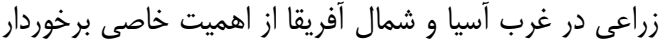

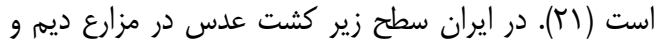

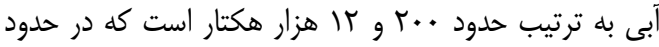

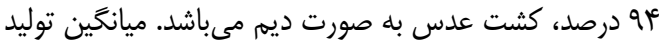

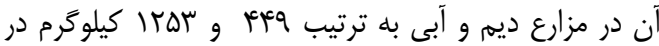

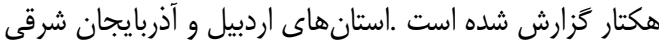

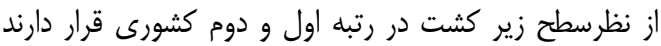

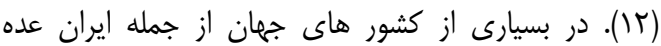

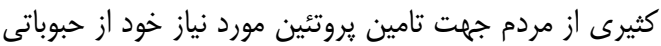

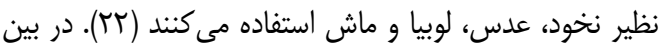

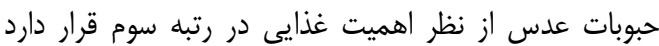

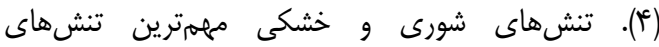

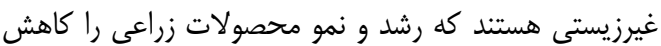

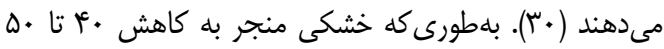

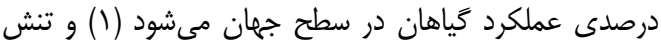

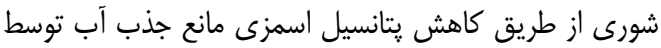

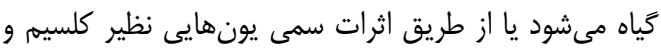

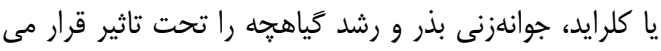

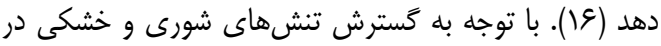

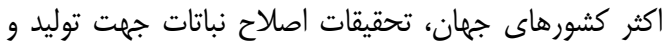

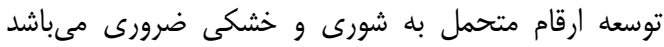

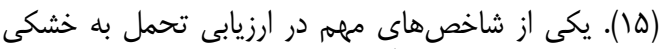

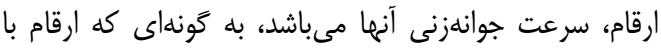

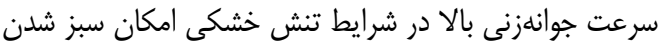

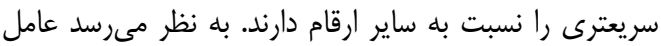


با توجه به مطالب فوق و اينكه تنش شورى و خشكى به

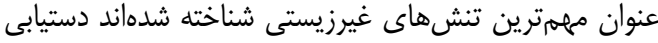

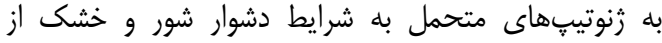

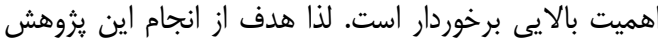

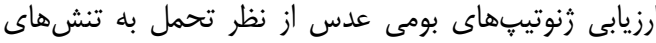

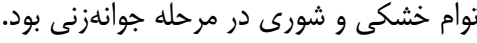

\section{مواد و روشها}

آزمايش حاضر با بكارگيرى س فاكتور (سطوح تنش

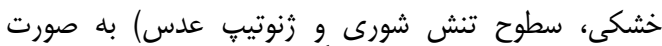

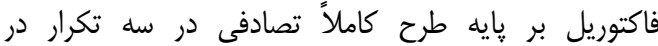

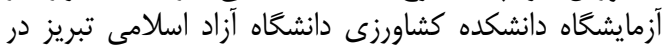

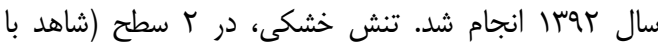

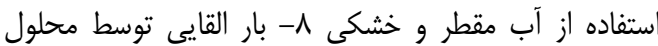

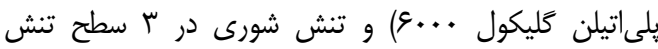

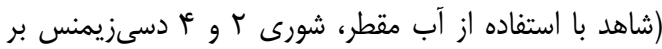

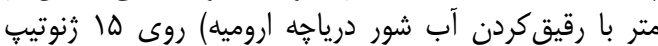
عدس (جدول () اعمال گرديد

درصد جوانهزدن را كاهش مىدهد. كاهش جوانهزنى و رشد

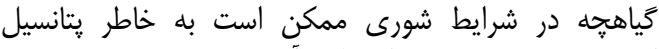

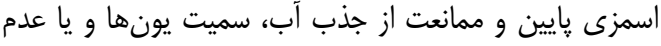

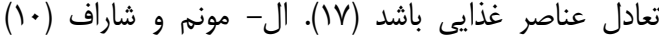

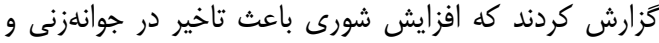

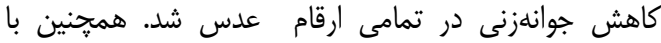

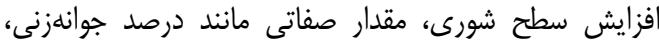

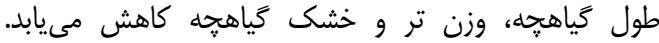

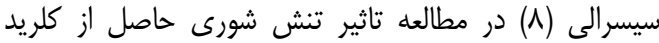

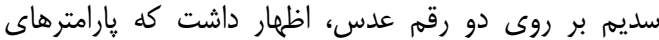

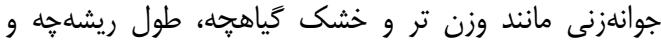

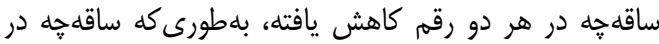

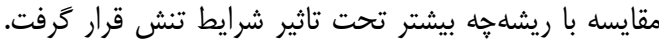

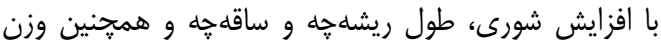

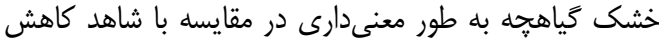

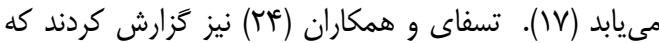

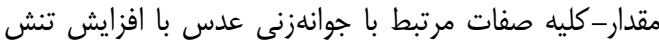

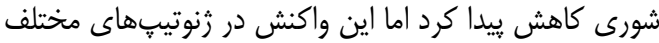

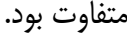

جدول 1- شماره و اسامى محل جمع آورى تودهاى بومى عدس

Table 1. Number and collection local of lentil landrace

\begin{tabular}{|c|c|c|c|c|c|}
\hline نام & شماره & نام & شماره & نام & شماره \\
\hline ورزقان & 11 & اردبيل & 9 & كانادا & 1 \\
\hline قره داغ دانه ريز & ir & هوراند & v & اهر & r \\
\hline علويق ورزقان & r & خاروانا & $\wedge$ & كليبر & r \\
\hline شاوى ورزقان & if & هوراند دانه ريز & 9 & كانادا دانه ريز & r \\
\hline ليملو اهر & 10 & داغ قيه قشلاقى & 1. & قره داغ & $\Delta$ \\
\hline
\end{tabular}

MSTAT-C مرديد. براى تجزيلهاى آمارى از نرم افزارهاى و SPSS استفاده شد.

\section{نتايج و بحث}

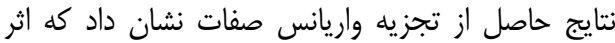

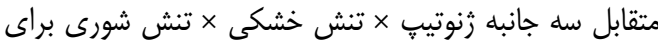

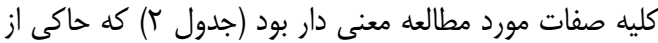

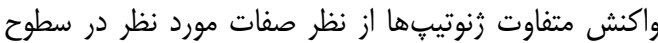

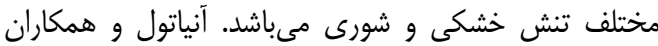

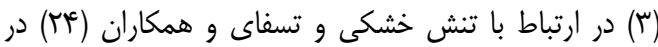

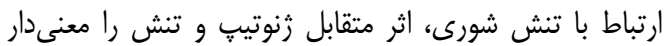

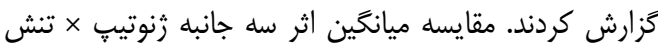

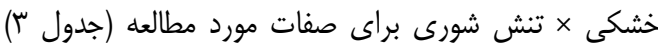

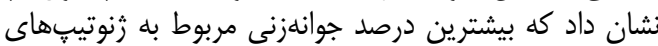

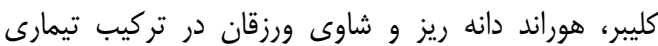

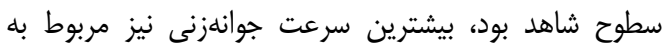

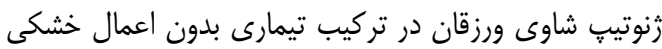

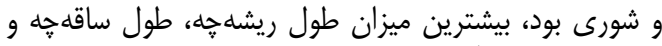

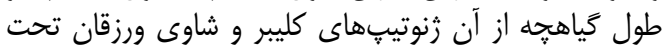

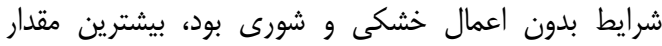

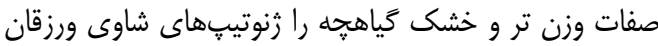

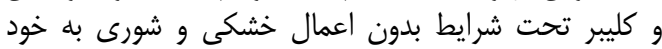

در اين آزمايش، اصول و روشهاى استاندارد ايستا به شيه

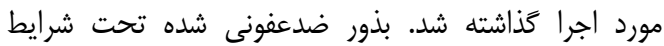

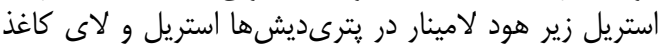

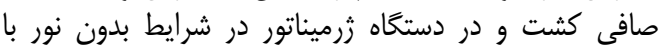
دماى افى

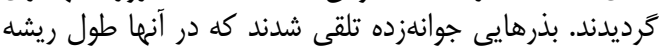

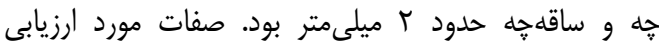

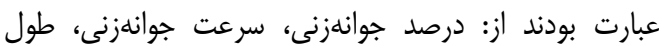

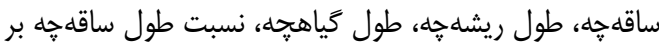

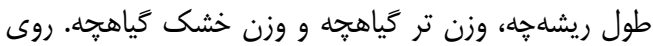

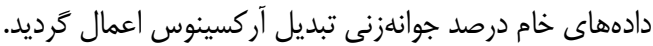

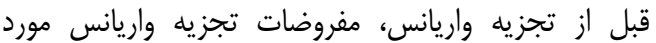

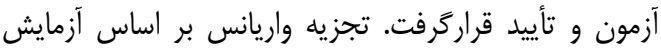

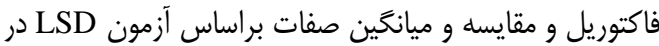

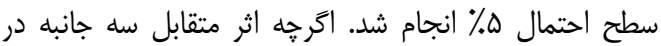

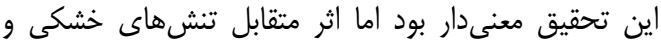

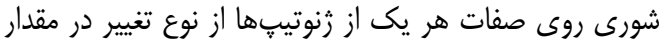

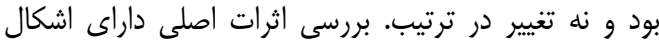

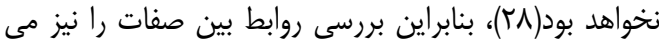

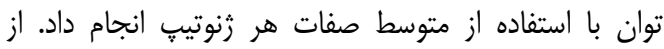

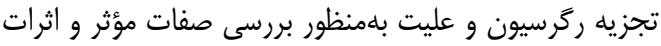
مستقيم و غيرمستقيم صفات مؤثر بر متغير وابسته استفاده 


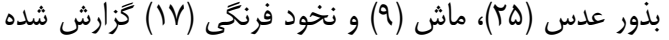

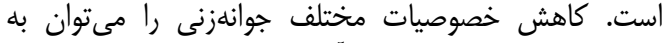

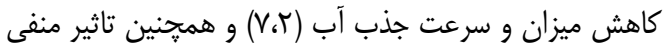

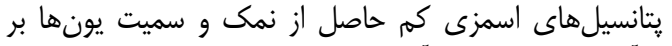

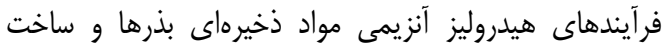

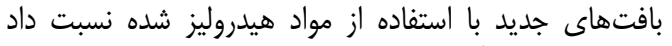

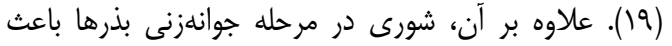

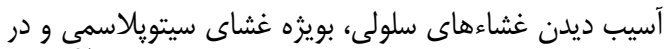
نتيجه آن افزايش تراوايى غشاءهاء

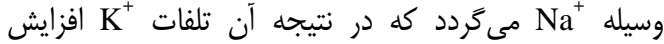

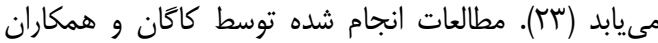

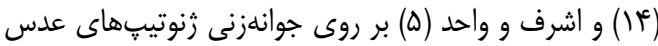

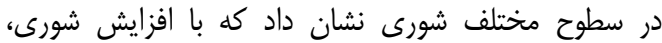

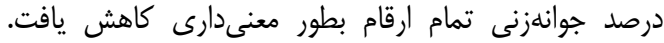

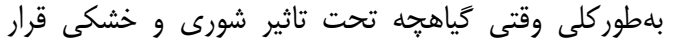

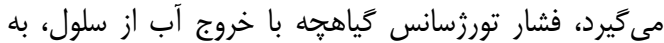

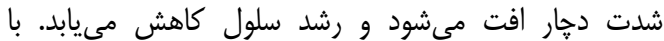

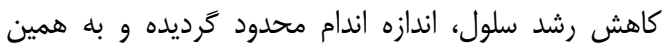

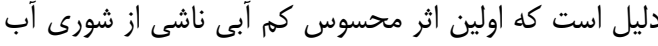

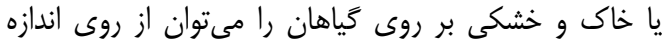

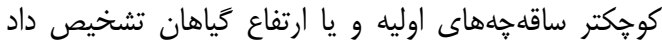

اختصاص دادند. بلهور كلى در تحقيق حاضر، با افزايش سطوح

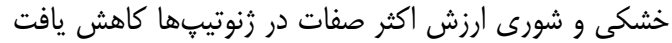

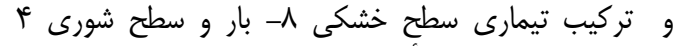

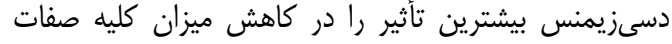

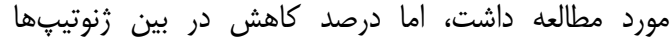

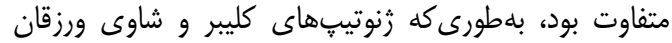

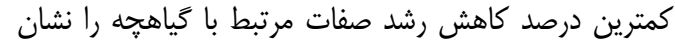

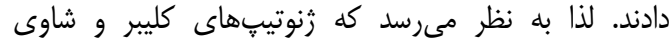

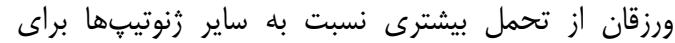

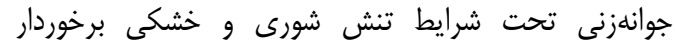

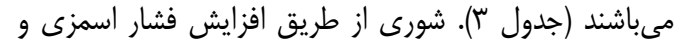

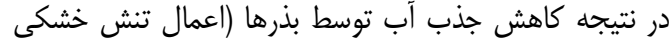

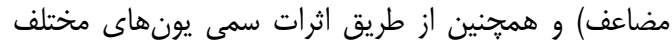

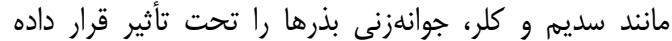

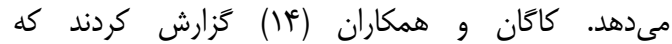

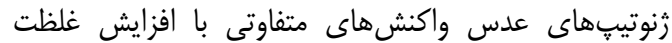

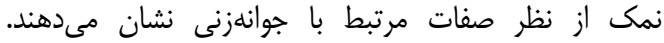

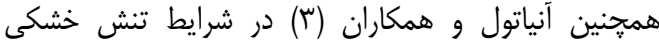

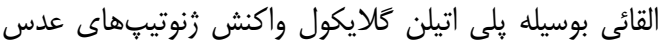

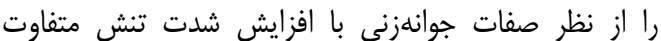

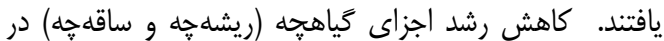
شرايط تنشهاى خشكى و شورى در ساير تحقيقات در مورد

جدول r- تجزيه واريانس صفات جوانهزنى زنوتيٍٍاى عدس در شرايط تنشهاى خشكى و شورى Table 2. Analysis of variance germination traits in lentil landrace genotypes under drought and salinity stress

\begin{tabular}{|c|c|c|c|c|c|c|c|c|c|}
\hline \multicolumn{8}{|c|}{ ميانكين مربعات } & \multirow[b]{2}{*}{ آزادى } & \multirow[b]{2}{*}{ منابع تغييرات } \\
\hline وزياهنه خشك & كَاهن تره & 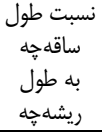 & طول كياهجه & طول ساقه & ريشهاجه & جوانهزنى & جوانهزنى & & \\
\hline $.1 \cdot \Delta q^{* \omega *}$ &.$/ N F T^{* B}$ &.$\cdot .1$ & $r \Psi V / 9 \Delta T^{*}$ & $q^{\mu} \uparrow \Delta / \Gamma \Delta S^{* * \omega}$ & $\Delta \Delta \& \backslash / 9 \Delta \digamma^{* \omega *}$ & $\Delta r / \cdot 9 q^{*}$ & $r \ldots \ldots$ & 1 & 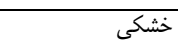 \\
\hline $.1 \cdot \Delta \gamma^{* * \omega}$ &.$/ 1 \Delta r^{* * *}$ &.$/ 11 \gamma^{* * a}$ & 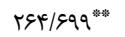 & 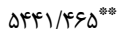 & VqIY/III" & $\mu \leftarrow / T M *$ & YIFT/VTL"*: & r & شورى \\
\hline$\cdot 1 \cdot 4 \Lambda^{* * *}$ & 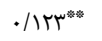 & 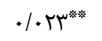 & $\mid T V / r \Lambda V^{* * *}$ & 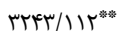 & 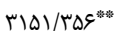 & $\Lambda V / \Delta T^{*}$ & $T r V \Psi / T V g^{* * *}$ & if & رنوتيّ \\
\hline$\cdot / \cdot \mathrm{V}^{*}$ &.$/ \cdot 1 \mathrm{~A}^{* * *}$ & $\cdot / \cdot V \Lambda^{* *}$ & $\mid \omega / \Delta \cdot 1^{* * *}$ & $\mid r \Delta / \Delta F \Lambda^{* * *}$ & $\Lambda \mid N / 9 \cdot 0^{* *}$ & $r / M .^{* *}$ & $F \Delta V / V / \Lambda^{* * *}$ & t & خشكي ×شورى \\
\hline.$/ . .1 V$ & $\cdot / \cdot r^{*}$ & r... & . & Ir/Ar.* & $\mid F / 9 T q^{* *}$ & ./AV9"*as & $\mid V / f V f^{f}$ & 15 & خشكى ×زثنوتيبٍ \\
\hline$\cdot 1 \cdot \cdot 1 \Lambda^{*}$ & $\cdot / \cdot r^{* * *}$ & $.1 . .9^{* * *}$ &.$/ 919^{* *}$ & 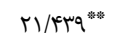 & $r \cdot 1 \cdot 9 \gamma^{*}$ &.$/ 94 r^{* 0.6}$ & $r \cdot / r T)^{* * *}$ & ru & شورى ×زنوتيب \\
\hline$\cdot 1 \cdot \cdot 1 \Lambda^{* * *}$ & $.1 . .19^{* * *}$ & $.1 . .9^{* * *}$ & $\cdot / 0 \cdot \mu^{*}$ & $r / / \Lambda q^{* * * * *}$ & $W / I V D^{* *}$ & 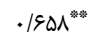 & $r \Delta / T V^{*}$ & ru & جشك شورى ×ثنوتيب \\
\hline $.1 . .1$ &.$/ .1$ &...$r$ & . Tat & 8/^9) & s/9. & $\cdot / T \Delta \Lambda$ & IF/TKY & 14 . & خطا \\
\hline$\Gamma / N \varepsilon$ & r/l & $r / \Delta$. & $r / v V$ & $r / v Q$ & $\% / .1$ & Q/IT & $g / r$. & --- & ضريب تغييرات (\%) \\
\hline
\end{tabular}


جدول بـ- مقايسه ميانگين صفات جوانهزنى در عدس تحت اثرات متقابل خشكى × شورى × زنوتيب Table 3. Comparison of germination traits mean in lentils under drought $\times$ salinity $\times$ genotype interactions

\begin{tabular}{|c|c|c|c|c|c|c|c|c|}
\hline 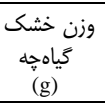 & 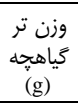 & $\begin{array}{c}\text { كول } \\
\text { كياهجه } \\
(\mathrm{cm})\end{array}$ & 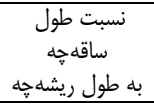 & طول ساقه جه & $\begin{array}{c}\text { ريش } \\
\text { (mm) } \\
\text { (m) }\end{array}$ & جوانهزنى & جوانهزنى (\%) & اثرات متقابل \\
\hline$\cdot / I T \Delta$ & $\cdot|| 9 \mid$ & $I r / T V$ & $1 / \cdot \Delta$ & $G N / D$ & $9 Q / / Q$ & אוr/N & 9. & $\mathrm{a}_{1} \times \mathrm{b}_{1} \times 1$ \\
\hline$\cdot / 1 \cdot V$ & אTM / & $I T / F$ & $V \cdot V$ & SF/IV & $\Delta q / V \Lambda$ & V/rq & سM/WM & $a_{1} \times b_{1} \times 2$ \\
\hline.$/ 491$ &. $\mid x+1$ & $T M / D I$ & $1 / \cdot \Delta$ & $11 \cdot / 8$ & $1 .+8 / 0$ & $\mid f / \& \lambda$ & 9. & $a_{1} \times b_{1} \times 3$ \\
\hline.$/ 118$ &.$/ 148$ & $I r / A V$ & $1 / \cdot 1$ & $99 / 9$ & GN/A & $N / \Delta \Delta$ & $\varepsilon$. & $a_{1} \times b_{1} \times 4$ \\
\hline$\cdot / T I F$ & - & $1 V / 09$ & $1 / \cdot r$ & $19 / F \Delta$ & $1 \varepsilon / \uparrow a$ & $1 r / 4 q$ & $\Lambda N / M F$ & $\mathrm{a}_{1} \times \mathrm{b}_{1} \times 5$ \\
\hline عسו/. & . MAr & $\mid F / \Delta T$ & $d \cdot r$ & טמאו & V)/q & $q / 4 V$ & $99 / 9 V$ & $a_{1} \times b_{1} \times 6$ \\
\hline سFl &.$/ 19$. & $15 / 99$ & $1 / .4$ & $V Q / A \Delta$ & $v^{c}$ & $1 . / 11$ & SE/SV & $a_{1} \times b_{1} \times 7$ \\
\hline פ & $\cdot /$ KND & 1N/Ta & W & $9 \pi / r$ & $q \cdot / r$ & $|r / 9|$ & $\Lambda T / M F$ & $a_{1} \times b_{1} \times 8$ \\
\hline س &.$/ 411$ & $19 / 0$ & س./ & $99 / \cdot \Delta$ & १८/৭ & IT/NA & 9. & $a_{1} \times b_{1} \times 9$ \\
\hline.$/ 194$ & ./TAD & $19 / V^{c}$ & $1 / \cdot \Delta$ & $\wedge \bowtie / \wedge$ & $\Lambda 1 / 9$ & IT/RT & $V \& / \& V$ & $a_{1} \times b_{1} \times 10$ \\
\hline $.1 \cdot 19$ &.$/ 119$ & $\mid K / \cdot F$ & $1 / \cdot e^{c}$ & $81 / 0$ & $\Delta N / Q \Psi$ & $s / V$ & سא/سם & $a_{1} \times b_{1} \times 11$ \\
\hline ./1rq & $\cdot / \Lambda \varepsilon$ & $\mid f / g$ س & r & $V F / \Delta$ & $v / v a$ & Q/AV & SE/SV & $a_{1} \times b_{1} \times 12$ \\
\hline.$/ 1 \Delta S$ & . /TTD & $\mid \Delta / \mathscr{Q}$ & r/ע & $V q / f a$ & VE/AD & $11 / \Delta 1$ & v. & $a_{1} \times b_{1} \times 13$ \\
\hline . & $\cdot 10 \cdot 1$ & $r \cdot / 9 \Lambda$ & $1 / \cdot f$ & $1.9 / 1$ & $1 \cdot r / 9$ & IV/RT & $q$. & $a_{1} \times b_{1} \times 14$ \\
\hline . MAr & $\cdot / T \Delta$ & $18 /{ }^{\prime} q$ & $1 / .9$ & $\Lambda F / T \omega$ & $\vee \vee / \Delta \Delta$ & $11 / 8$ & $V \& / \& V$ & $a_{1} \times b_{1} \times 15$ \\
\hline .1 .94 &.$/ 119$ & IT/ET & $1 / .9$ & $9 F / 10$ & $\varepsilon$ & $N \cdot r$ & Q. & $\mathrm{a}_{1} \times \mathrm{b}_{2} \times 1$ \\
\hline$\cdot 1 \cdot 1$ & r.9. & $11 / r r$ & I/T & $\Delta Q / M$ & $\Delta H / F V$ & s/V^ & r. & $a_{1} \times b_{2} \times 2$ \\
\hline$\cdot / 4 \cdot 1$ & ./TqF & $|N| \cdot V$ &.$/ 99$ & $9 \cdot / 1$ & $9 \cdot / 8$ & IT/NE & سא/WV & $a_{1} \times b_{2} \times 3$ \\
\hline.$/ \cdot 1 \varepsilon$ & $.11 \cdot 9$ & $\mid w / \cdot V$ & $1 / .9$ & sV/Tu & هT/ & $N / E^{\prime} \Delta$ & سז/Wט & $\mathrm{a}_{1} \times \mathrm{b}_{2} \times 4$ \\
\hline$\cdot / 1 \Delta \Delta$ & . & $\mid 8 / \cdot 1$ & $1 /$. & $1 \cdot / ז \Delta$ & $\Lambda \cdot / \pi \Delta$ & $11 / \Delta \Lambda$ & SG/9V & $a_{1} \times b_{2} \times 5$ \\
\hline$\cdot / 1 \cdot \Delta$ & . ITE & Ir/TV & $1 / .4$ & SV/FD & 99 & N/q & $\varepsilon$. & $\mathrm{a} 1 \times \mathrm{b} 2 \times 6$ \\
\hline سו/ & . lift & سואוא & $1 / \cdot f$ & $V T / r$ & 99 & N/AT & c. & $a_{1} \times b_{2} \times 7$ \\
\hline.$/ I V T$ & - / Trq & $18 / 94$ &.$/ 98$ & N & N & $11 / \lambda f$ & GE/SV & $\mathrm{a}_{1} \times \mathrm{b}_{2} \times 8$ \\
\hline$\cdot / \backslash \wedge \vee$ & $\cdot / T H$ & $|V / T|$ &.$/ 90$ & $\Lambda F / r$ & $M / V \Delta$ & $11 / V \pi$ & سא/WV & $\mathrm{a}_{1} \times \mathrm{b}_{2} \times 9$ \\
\hline גזו/. &.$/ T I f$ & $|\Delta / 9|$ & $1 / \cdot r$ & VN/AD & $V V / r \Delta$ & $11 / T V$ & Gq/9V & $a_{1} \times b_{2} \times 10$ \\
\hline$\cdot 1 \cdot v^{m}$ & $\cdot 1 \cdot 1 \Delta$ & 1.19 & I/T & $\Delta S / T T$ & $\mathrm{rq} / \mathrm{V}$ & $9 / 19$ & $f$ & $a_{1} \times b_{2} \times 11$ \\
\hline .11 .9 & ./ITV & $1 \pi / 4$ & r & GN/T & $G \Delta / V \Delta$ & $q / \Delta T$ & 9. & $\mathrm{a}_{1} \times \mathrm{b}_{2} \times 12$ \\
\hline.$/ 119$ &.$/ 1 f q$ & If/Ta & $1 / \cdot 1$ & $V F / v a$ & GN/V & $11 / \cdot V$ & $98 / 9 \mathrm{~V}$ & $a_{1} \times b_{2} \times 13$ \\
\hline.$/ T I D$ & זוז/. & $11 / 9$ & $1 / \cdot f^{4}$ & $q \varepsilon / f \Delta$ & $Q T / \Delta D$ & Ir/ar & $V \& / \& V$ & $a_{1} \times b_{2} \times 14$ \\
\hline.$/ I r A$ &.$/ 119$ & $\mid f / \& \Delta$ & $1 / 11$ & $\mathrm{~W} / \mathrm{m}$ & $99 / 10$ & $|1 / r|$ & $98 / 9 \mathrm{~V}$ & $a_{1} \times b_{2} \times 15$ \\
\hline$\cdot 1 \cdot 11$ & .1 .94 & $q / r V$ & $1 / 11$ & $r q / T \omega$ & $F F / T \Delta$ & V/Ar & rw/Tre & $a_{1} \times b_{3} \times 1$ \\
\hline .1 .90 & $\cdot / \cdot v^{\mu}$ & N/AF & $1 / 14$ & $r e / V$ & $|f| / \& D$ & S/OT & f. & $a_{1} \times b_{3} \times 2$ \\
\hline.$/ 19$. &.$/ T V K$ & $10 / 98$ & سו/ & $\Lambda F / \Lambda$ & $V F / \Lambda$ & $|r / F|$ & سא/WV & $a_{1} \times b_{3} \times 3$ \\
\hline$\cdot 1 \cdot v r$ & $\cdot / \cdot 18$ & $1 . / 1 T$ & $1 / .9$ & $\Delta T / A \Delta$ & $\boldsymbol{F} N / \mu$ & N/HF & Fe/ $9 \mathrm{~V}$ & $a_{1} \times b_{3} \times 4$ \\
\hline$\cdot / 10$ & שוT/ & $\mid F / \Delta F$ & $1 / .9$ & $V \omega / \Lambda$ & $\Phi q / \Delta \Delta$ & 1.191 & $99 / 9 \mathrm{~V}$ & $\mathrm{a}_{1} \times \mathrm{b}_{3} \times 5$ \\
\hline .1 .98 & .11 .9 & $11 / T r$ & $1 / 1 r$ & $\Delta q / f$ & $\Delta T / V Q$ & N/ET & $\Delta S / S V$ & $a_{1} \times b_{3} \times 6$ \\
\hline .11 .9 &.$/ I r A$ & $1 \pi / 1 f$ & $1 / 1$. & GN/৭D & $g r / f$ & N/AT & $\Delta F / F V$ & $a_{1} \times b_{3} \times 7$ \\
\hline . Mat & . ITHA & $\mid \Delta / \cdot 1$ & $1 / 1$. & VN/AD & $V I / r$ & $11 / T \Delta$ & $89 / 9 \mathrm{~V}$ & $a_{1} \times b_{3} \times 8$ \\
\hline.$/ 189$ & . / TFq & $10 / T \&$ & سו & 11 & $\checkmark \backslash / \Delta \Delta$ & $11 / 1 r$ & $V \cdot$ & $\mathrm{a}_{1} \times \mathrm{b}_{3} \times 9$ \\
\hline |II|. &.$/ 19$. & $15 / 99$ & $1 / 14$ & $V \pi / q$ & 99 & $1 . / 91$ & SE/SV & $a_{1} \times b_{3} \times 10$ \\
\hline$\cdot / \cdot \Delta$ & .1 .94 & $N \cdot r$ & $1 / R \Delta$ & $P+/ D$ & سת & $\Delta / M$ & r. & $a_{1} \times b_{3} \times 11$ \\
\hline $.1 .9 V$ & $\cdot 11 \cdot 1$ & II/Tr & $1 / 1 r$ & $\Delta Q / \wedge \Delta$ & $\Delta F / \Gamma$ & $9 / 19$ & $\Delta F / G V$ & $a_{1} \times b_{3} \times 12$ \\
\hline.$/ 114$ & سזו/. & IT/KT & $1 / 19$ & $V T / \cdot D$ & ST/1 & 1./ & $98 / 9 V$ & $a_{1} \times b_{3} \times 13$ \\
\hline$\cdot / t \cdot r$ & ./rqf & IE/AV & $1 / 19$ & १)/АЬ & VE/A & $\mid r / \Delta \Lambda$ & سא/سV & $a_{1} \times b_{3} \times 14$ \\
\hline.$/ 14$ & $\cdot / 1 \Delta S$ & $115 / 09$ & I/T & VT & q & $1 \cdot / V 1$ & GE/\&V & $a_{1} \times b_{3} \times 15$ \\
\hline$\cdot 1 \cdot 0$ & $\cdot 1 \cdot \Delta$ & ./AT & $\cdot / \cdot V$ & $F / T H$ & $F / T \Delta$ & $\cdot|\wedge|$ & 91.9 & LSD $\% \bowtie$ \\
\hline
\end{tabular}




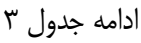

Table 3 Continue

\begin{tabular}{|c|c|c|c|c|c|c|c|c|}
\hline $\begin{array}{c}\text { وزياهجه خشك } \\
\text { (g) }\end{array}$ & 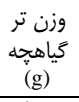 & $\begin{array}{c}\text { طي } \\
\text { طياهج } \\
(\mathrm{cm})\end{array}$ & 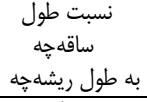 & $\begin{array}{c}\text { سوله } \\
\text { (mm) } \\
\text { (mm } \\
\end{array}$ & $\begin{array}{l}\text { طول } \\
\text { ريشه } \\
(\mathrm{mm}) \\
\end{array}$ & جوانهزنى & جوانهزنى (٪) & اثرات متقابل \\
\hline .1 .99 & ( & $11 / 91$ & $1 / .9$ & GI/VQ & $\Delta \mathrm{N} / \cdot \mathrm{\Delta}$ & $V / 19$ & $F \& / 9 V$ & $\mathrm{a}_{2} \times \mathrm{b}_{1} \times 1$ \\
\hline$\cdot / \cdot \Delta \Delta$ & $.1 .9 V$ & IVT & $1 / \cdot 0$ & $\Delta 9 / 1$ & $\Delta S / \backslash \Delta$ & $g / V \mid$ & $r$ & $a_{2} \times b_{1} \times 2$ \\
\hline . & $\cdot / \mu$ & W/TF &.$/ 99$ & $q \cdot / V \Delta$ & १1/ब८ & $1 \% / 19$ & $V G / 9 V$ & $\mathrm{a}_{2} \times \mathrm{b}_{1} \times 3$ \\
\hline .1 .9 &.$/ 114$ & $\mid r / 9 V$ & $1 / .4$ & $G \% / \& D$ & $9 \pi / \cdot 0$ & $N / \mu \Lambda$ & $19 / 9 V$ & $a_{2} \times b_{1} \times f^{f}$ \\
\hline$\cdot / \mid \Delta \wedge$ & . & $19 / \pi F$ & $1 / \cdot$. & $\wedge 1 / \kappa^{\epsilon}$ & 11 & $11 / 91$ & v. & $\mathrm{a}_{2} \times \mathrm{b}_{1} \times 5$ \\
\hline.$/ 11$ & . Irfe & $1 \% / \Delta$ & $1 / \mu$ & SN/VQ & se/ra & $N / \Lambda$ & $\Delta F / G V$ & $\mathrm{a}_{2} \times \mathrm{b}_{1} \times 6$ \\
\hline.$/ 110$ &.$/ 148$ & $1 F / 10$ & $1 / \cdot 4$ & $V T / \pi \Delta$ & $99 / 10$ & N/VT & ك Tr/ & $\mathrm{a}_{2} \times \mathrm{b}_{1} \times 7$ \\
\hline.$/$ V & . /TAF & $\mid V / 1$ &.$/ 90$ & $\Lambda \mu / v$ & $\Lambda V / r$ & 11/9 & v. & $\mathrm{a}_{2} \times \mathrm{b}_{1} \times 8$ \\
\hline.$/ 191$ & $\cdot / T V A$ & $\mid V / A V$ &.$/ 94$ & $\Lambda F / \& \Delta$ & $9 \cdot 1 \cdot 0$ & II/Ar & $V \xi \mid q V$ & $\mathrm{a}_{2} \times \mathrm{b}_{1} \times 9$ \\
\hline.$/ 1 F A$ & $\cdot / T \mid S$ & $1 \Delta / V \Delta$ & $1 / . r$ & $\mathrm{~V} / \mathrm{V}$ & $\mathrm{V} / \mathrm{A}$ & $11 / \pi 1$ & $98 / 9 \mathrm{~V}$ & $a_{2} \times b_{1} \times 10$ \\
\hline.$/ \cdot \Delta V$ & $.1 \cdot 19$ & $1.10 \Delta$ & $1 / \cdot 0$ & $\Delta F / \cdot V$ & $\Delta / / F^{2} V$ & $\varepsilon / \pi \Delta$ & r. & $a_{2} \times b_{1} \times 11$ \\
\hline$\cdot 11 \cdot 1$ & r & g & $1 / \mu$ & SV/AD & $9 \Delta / v \Delta$ & $9 / 41$ & $\Delta F / G V$ & $a_{2} \times b_{1} \times 12$ \\
\hline . ITr & . / IQT & $1 F / T e$ & $1 / 11$ & $V \Delta$ & $S V / \Delta D$ & $11 / 10$ & $98 / 9 \mathrm{~V}$ & $a_{2} \times b_{1} \times 13$ \\
\hline$\cdot / \pi r$ & (אוץ/. & W/AT & $1 / . r$ & $90 / T$ & $9 \% / 0$ & $\pi$ & $\Lambda$. & $a_{2} \times b_{1} \times 14$ \\
\hline ( & .1198 & $|f / \lambda|$ & $1 /$. & W/VD & $V \cdot / r$ & $11 / T V$ & $98 / 9 \mathrm{~V}$ & $a_{2} \times b_{1} \times 15$ \\
\hline$\cdot / \cdot v e$ & $\cdot 1 \cdot M$ & N/AV & $1 / 11$ & $\mid \varphi / V$ & et & s/va & $r / \mu f$ & $\mathrm{a}_{2} \times \mathrm{b}_{2} \times 1$ \\
\hline$\cdot / \cdot \Delta \Delta$ & .1 .94 & $N / 1$ & $1 / .9$ & $r 1 / \Lambda$ & $r q / r$ & $8 / \Delta$ & r. & $\mathrm{a}_{2} \times \mathrm{b}_{2} \times 2$ \\
\hline$\cdot / \backslash \Lambda \mid$ & $\cdot / T \Delta T$ & $\mid F / \wedge \Delta$ & $1 / I V$ & $1 \cdot 1 \cdot \Delta$ & $9 N / K^{\circ}$ & $\mid r / T \Lambda$ & v. & $\mathrm{a}_{2} \times \mathrm{b}_{2} \times 3$ \\
\hline 1.94 &.$/ \cdot v g$ & $q / r q$ & $1 / 1$. & FN/FD & $\mu \varphi / T$ & V/Ar & FT/MF & $\mathrm{a}_{2} \times \mathrm{b}_{2} \times 4$ \\
\hline . Mft & $\cdot / t \cdot t$ & $\mid r / F V$ & $1 / .9$ & $v \cdot / r \omega$ & $94 / \pi$ & $1 . / \mathrm{VA}$ & q. & $a_{2} \times b_{2} \times 5$ \\
\hline 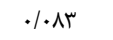 & .1 .98 & $1 . / \% F$ & $1 / 1$. & $\Delta F / I Q$ & $r q / r$ & $\Lambda / \Lambda$ & $\Delta \Gamma / T F$ & $\mathrm{a}_{2} \times \mathrm{b}_{2} \times 6$ \\
\hline .1 .9 &.$/ 111$ & $15 / .9$ & $1 / \cdot 1$ & א & $\Delta \mathrm{V} / \mathrm{q}$ & $N / \widetilde{\mu}$ & $\Delta \Gamma / \mu F$ & $\mathrm{a}_{2} \times \mathrm{b}_{2} \times 7$ \\
\hline$\cdot / 1 \Delta F$ & $\cdot / K I F$ & $1 \% / v \wedge$ & /1. & $V \pi / r \Delta$ & $\varepsilon \Delta / \kappa \Delta$ & $11 / \cdot r$ & 9. & $\mathrm{a}_{2} \times \mathrm{b}_{2} \times 8$ \\
\hline .1199 & . & $15 / .9$ & $1 / .9$ & $V \pi / \Delta$ & $9 \mathrm{~V} / \mathrm{F}$ & $11 / .9$ & v. & $\mathrm{a}_{2} \times \mathrm{b}_{2} \times 9$ \\
\hline . IMF & . MAT & $1 \% / M$ & $1 / 1$ & 99 & sT/VQ & 1. & 9. & $\mathrm{a}_{2} \times \mathrm{b}_{2} \times 10$ \\
\hline תז./. &.$/ . \Delta T$ & $V / . \mu$ & $1 / r 1$ & $\Gamma / \uparrow \Lambda$ & M & $\Delta / 89$ & f. & $\mathrm{a}_{2} \times \mathrm{b}_{2} \times 11$ \\
\hline$\cdot / \cdot \Lambda \mu$ & $\cdot / 1 \cdot r$ & $1 \cdot / \Delta T$ & $1 / \cdot V$ & $\Delta F / \Delta \omega$ & $\Delta \cdot / 9$ & $N / \Delta S$ & $\Delta$. & $\mathrm{a}_{2} \times \mathrm{b}_{2} \times 12$ \\
\hline$\cdot / 1 \cdot \Delta$ & ./1rq & $|r / 9|$ & $1 / \cdot 1$ & $9 \Delta / \kappa \Delta$ & $9 \cdot 19$ & $1 . / 11$ & $\Delta F / q V$ & $\mathrm{a}_{2} \times \mathrm{b}_{2} \times 13$ \\
\hline.$/ 19$ & $\cdot|r V|$ & $18 / 09$ & $1 / 11$ & $19 / \wedge$ & VE/I & $1 T / \pi r$ & $V \pi / r \mu$ & $\mathrm{a}_{2} \times \mathrm{b}_{2} \times 14$ \\
\hline$\cdot / 11 f$ & $\cdot|| Q \mid$ & $1 \% / 94$ & $1 / \cdot 1$ & $q V / T \Delta$ & $g \pi / 10$ & $1 \cdot / \Delta T$ & 9. & $\mathrm{a}_{2} \times \mathrm{b}_{2} \times 15$ \\
\hline .1 .94 & $\cdot 1 \cdot V^{e}$ & $N \cdot r$ & $1 / \cdot 1$ & $F \mid / V D$ & $r / / F \Delta$ & $9 / 9 \Lambda$ & $F \Psi / \mu F$ & $\mathrm{a}_{2} \times \mathrm{b}_{3} \times 1$ \\
\hline .1 .48 & $\cdot 1 \cdot \Delta V$ & V/rr & $1 / \cdot V$ & $\mathrm{TV} / \mathrm{QD}$ & $\Gamma / \Gamma \Delta$ & G/FT & FT/KF & $\mathrm{a}_{2} \times \mathrm{b}_{3} \times 2$ \\
\hline$\cdot / 1 \mathrm{VI}$ & וזוא. & $|f / v|$ & $1 / .9$ & VQ/VD & $\checkmark 1 / \Gamma \Delta$ & 11 & 9. & $\mathrm{a}_{2} \times \mathrm{b}_{3} \times 3$ \\
\hline $.1 \cdot \Delta \xi$ & .1 .90 & $9 / v r$ & $1 / \cdot V$ & $\Delta \cdot / \% \omega$ & $+8 / 9$ & $V / F 1$ & Q. & $\mathrm{a}_{2} \times \mathrm{b}_{3} \times 4$ \\
\hline . IMf &.$/ 19$ & $1 \% / 90$ & $1 / .9$ & $99 / 90$ & $9 \pi / \Lambda$ & $1 \cdot / r V$ & ת & $\mathrm{a}_{2} \times \mathrm{b}_{3} \times 5$ \\
\hline$\cdot / \cdot v^{e}$ &.$/ 1 r$ & $1 . / \kappa \Lambda$ & $1 / \cdot \mu$ & $\Delta \Gamma / 9 D$ & $\Delta 1 / 1$ & $N / 1$ & $\Delta S / G V$ & $\mathrm{a}_{2} \times \mathrm{b}_{3} \times 6$ \\
\hline$\cdot / \cdot \Lambda \mu$ & .1 .94 & $11 / M$ &.$/ 91$ & $\Delta q$ & $\Delta Q / \Lambda$ & $\Lambda / \Lambda$ & $\Delta \Psi / M F$ & $a_{2} \times b_{3} \times 7$ \\
\hline . IFA & $\cdot / T \cdot T$ & $\mid r / g 4$ & $1 / \cdot 0$ & $89 / \wedge \Delta$ & $99 / 0$ & $1 . / \Delta T$ & & $\mathrm{a}_{2} \times \mathrm{b}_{3} \times 8$ \\
\hline$\cdot / 1 \Delta \Delta$ &.$/ T I \Delta$ & $1 \% / .9$ & $1 / . \mu$ & $\checkmark 1 /{ }^{\prime} \Delta$ & $s q / r \Delta$ & $1 . / \mathrm{VA}$ & v. & $\mathrm{a}_{2} \times \mathrm{b}_{3} \times 9$ \\
\hline . / &.$/ 19 V$ & $\mid r / \Lambda$ & $1 / \cdot 0$ & $9 \Delta / V$ & Gr/r & 1. & 9. & $a_{2} \times b_{3} \times 10$ \\
\hline.$/ \cdot T V$ & $\cdot 1 \cdot 4 \Delta$ & $\Delta / \& \Lambda$ & $1 / \pi \Delta$ & $\Gamma / T V$ & $r \Delta / \Delta$ & $\Delta / r^{\prime} q$ & $19 / 9 V$ & $a_{2} \times b_{3} \times 11$ \\
\hline$\cdot / \cdot V \Delta$ & $\cdot 1 \cdot 1 \Delta$ & $1 . / 49$ & $1 / \cdot r$ & $\Delta r / q$ & Q) & N/FT & $\Delta \Gamma / \mu F$ & $a_{2} \times b_{3} \times 12$ \\
\hline .1 .90 & $\cdot 11 \cdot \Delta$ & $11 / 94$ & $1 / 19$ & $9 \pi / 9$ & $\Delta \mu / \bar{G}$ & १/१८ & $\Delta \& / \overline{Q S V}$ & $a_{2} \times b_{3} \times 13$ \\
\hline . /NAr & . & $10 / 19$ & $1 / .9$ & $V Q / r \Delta$ & $V Y / S$ & $11 / \pi \mu$ & v. & $a_{2} \times b_{3} \times 14$ \\
\hline$\cdot 11 \cdot 1$ &.$/ 110$ & $1 \Gamma / \pi \Delta$ & $1 / \cdot 1$ & $g \% / r$ & $\Delta q / T \Delta$ & $1 . / 1 \pi$ & 9. & $\mathrm{a}_{2} \times \mathrm{b}_{3} \times 15$ \\
\hline .1 .0 & $\cdot 1 \cdot \Delta$ & - /Ar & $.1 \cdot V \pi$ & F/T & $\tau / \pi \Delta$ & $\cdot|\Lambda|$ & $8 / .9$ & LSD $\% \Delta$ \\
\hline
\end{tabular}

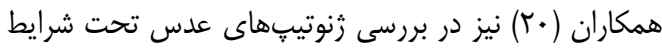

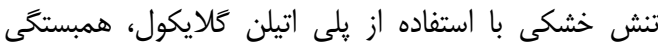

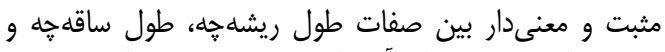

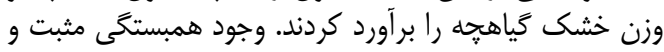

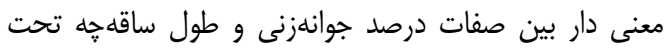

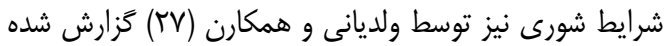

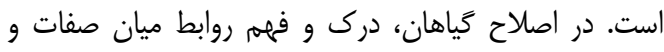

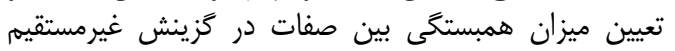

اگرجه اثر متقابل سه جانبه در اين تحقيق معنى دار بود اما

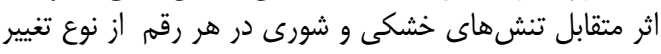

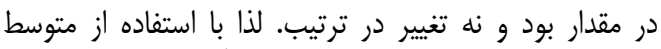

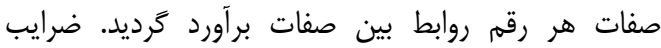

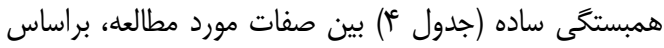

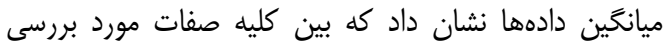

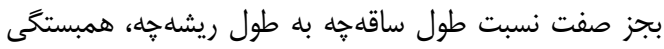

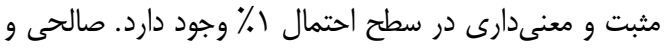


خه و نسبت طول ساقه קه به طول ريشه

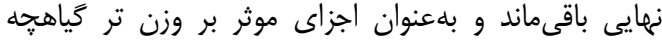
محسوب شدند:

$\mathrm{Y}=-1 / 199+\cdot / \cdot 11 \mathrm{X}_{1}-\cdot / \cdot 11 \mathrm{X}_{2}+\cdot / 1 \mathrm{VV} \mathrm{X}_{3}$

كه در اين مدل X

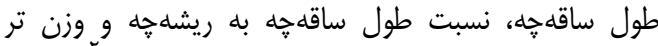

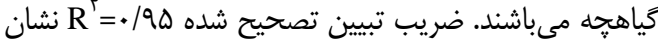

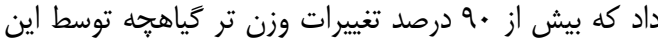

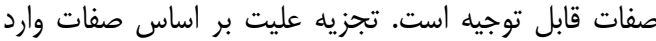

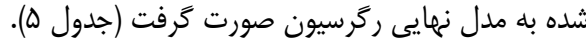

براى صفاتى كه به آسانى قابل اندازهگيرى نمىباشند، يا

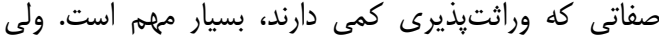

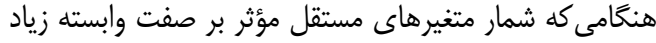

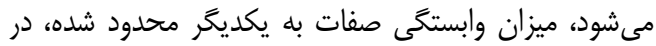

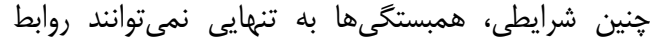

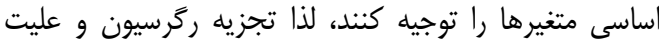

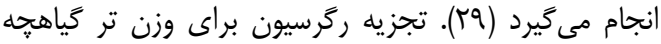

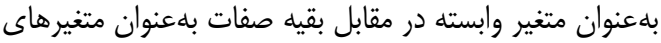

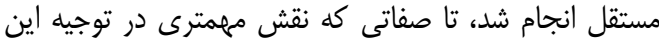

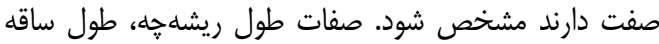

جدول fأ- ضرايب همبستخى ساده بين صفات جوانهزنى در زنوتيِهاى عدس تحت شرايط تنش خشكى و شورى Table 4. Simple correlation coefficient between germination traits in lentil genotypes under drought and salinity stress

\begin{tabular}{|c|c|c|c|c|c|c|c|}
\hline وزن تر كياهُه & 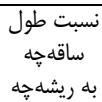 & 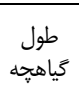 & ساقه קه & ريشه קهل & جوانهزعن & جوانهزنى & \\
\hline & & & & & & $\cdot / 9 \Lambda^{* * 2}$ & سرعت جوانهزنى \\
\hline & & & & & 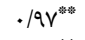 &.$/ 9 V^{* * 4}$ & طول ريشه קه \\
\hline & & & &.$/ 99^{* * *}$ &.$/ 94^{* a t a n}$ &.$/ 9 V^{* *}$ & طول ساقه \\
\hline & & &.$/ 99^{* \infty}$ & $.199^{\text {wh }}$ & 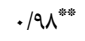 &.$/ 9 V^{* *}$ & طول كياهجه \\
\hline & &.$- / 4 r$ &.$- / 4 T$ &.$- / \Delta T^{* \prime}$ & -. &.$- / 44$ & نسبت طول ساقه جه به ريشهجه \\
\hline & $-\cdot / \% q$ & $.199^{* * 1+4}$ & $.199^{* * 3}$ & $.199^{m *}$ & 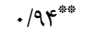 & . $/ 9 r^{*: m}$ & وزن تر كَاهجه \\
\hline $.199^{* * * 4}$ & $-. / 4 \mid$ &.$/ 9 Y^{* w a t}$ &.$/ 9 V^{* * 3}$ &.$/ 9 V^{* m}$ &.$/ 99^{4 * k n}$ &.$/ 99^{* m}$ & وزن خشك كَياهجه \\
\hline
\end{tabular}

جدول هـ تجزيه عليت وزن تر كَاهجه زنوتيڤهاى عدس تحت شرايط تنش خشكى و شورى Table 5. Path analysis of seedling fresh weight in lentil genotypes under drought and salinity stress

\begin{tabular}{|c|c|c|c|c|c|}
\hline \multirow{2}{*}{ همبستكى } & \multicolumn{3}{|c|}{ اثر غيرمستقيهم از طريق } & \multirow{2}{*}{ اثر مستقيم } & \multirow{2}{*}{ صفات وارد شده به مدل } \\
\hline & نسبت طول ساقه & طول ساقه & طول ريشه קه & & \\
\hline.$/ 98^{* * m}$ & $-\cdot / 19$ & $-1 / \sqrt{ } 9$ & --- & T/QT & طول ريشه خه \\
\hline.$/ 98^{* * 3}$ &.$- / 19$ & --- & $r / q$. & $-1 / \mathrm{V}$ & طول ساقه خه \\
\hline.$- / \% q$ & --- & $\cdot$ IVE & 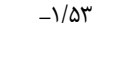 & $\begin{array}{l}. / 4 r \\
. / 19\end{array}$ & 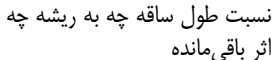 \\
\hline & & & & .917 & ***: بعنى مادره در سطح احتمال 1\% \\
\hline
\end{tabular}

بلمطوركلى در تحقيق حاضر، با افزايش سطوح خشكى و واهن

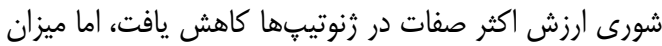

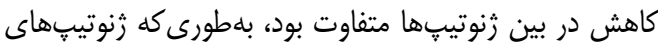

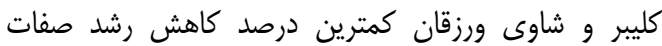

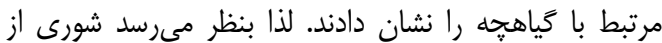

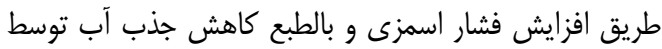

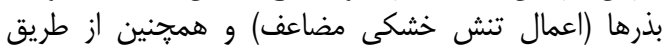

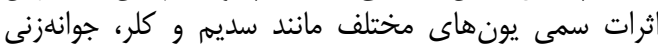

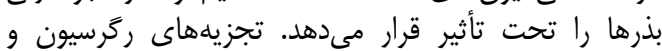

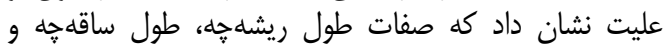

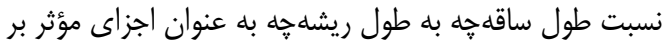

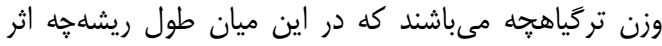

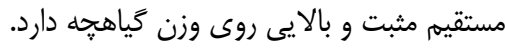

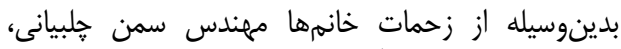

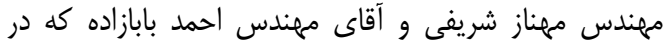
اجراى اين تحقيق همكارى صميمانهاى داشتند قدردئ فردانى مىشود.

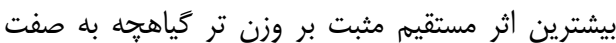

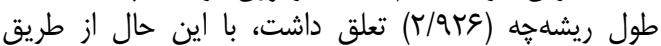

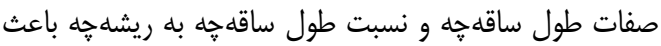

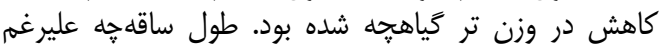

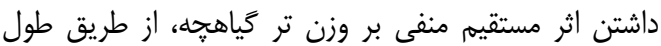

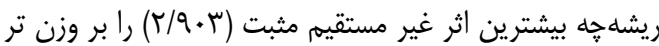

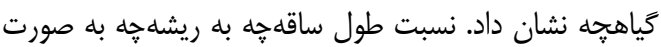

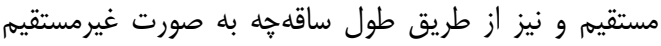

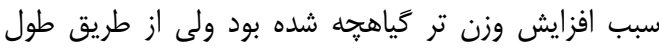

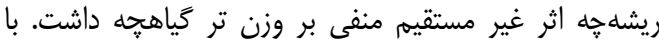

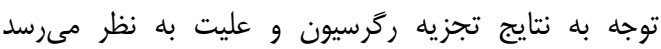

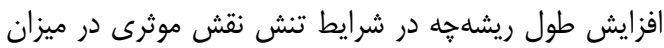

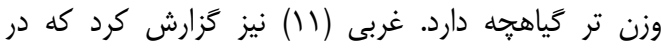

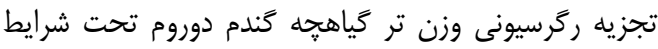

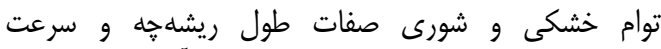

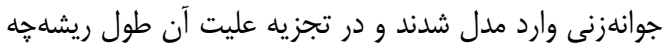

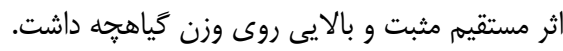


er واكنش جواننزنى و صفات كَياهجهاى زُوتيبهاى بومى عدس به تنش خشكى و شورى و

1. Ahmad, F., P. Gore and J. Croser. 2005. Chickpea (Cicer arietinum L.). In Genetic Resources, Chromosome Engineering and Crop Improvement - Grain Legumes, Vol. 1 (Singh, R. and Jauhar, P., eds). USA: CRC Press, $185-214 \mathrm{pp}$.

2. Allen, S.G., A.K. Dobrenz and P.G. Bartels. 1986. Physiological response of salt tolerant and non tolerant alfalfa to salinity during germination. Crop Science, 26: 1004-1008.

3. Aniatul-Haq, H., R. Vamil and R.K. Agnihotri. 2012. Effect of osmotic stress (PEG) on germination and seedling survival of lentil (Lens culinaris M.). Research Journal of Agricultural Sciences, 1: 201-202.

4. Asadi Chleshtari, C., A.E. Hasanzadeh Gorottapeh and A. Fayaz Moghaddam. 2007. Study of drought tolerance indices in lentil landraces in west Azarbayjan. Journal of Agricultural Sciences and Natural Resources, 13: 79-80 (In Persian).

5. Ashraf, M. and A. Waheed. 1990. Screening of local exotic of lentil (Lens Culinaris M.) for salt tolerance at two growth stages. Plant and Soil, 128: 167- 176.

6. Bakhtiar, B. and A. Shaykra. 1990. Drought tolerance in lentil. II differential genotypes response to drought. Journal of Agricultural Research, Lahore, 28:117-126.

7. Chadho, K. and G. Rajender. 1995. Advance in horticulture medicinal and aromatic plants. Maldorta Publisher. New Delhi, 11 pp.

8. Cicerali, I.N. 2004. Effect of salt stress on antioxidant defense systems of sensitive and resistant cultivars of lentil (Lens culinaris M.). M.Sc. Thesis, 90 pp.

9. De, F. and R.K. Kar. 1994. Seed germination and seedling growth of mung bean (Vigna radiate) under water stress included by PEG-6000. Seed Science and Technology, 23: 301-304.

10. El- Monem, A. and M. Sharaf. 2008. Tolerance of five genotypes of lentil to NaCl-salinity stress. New York Science Journal, 1: 70-80 pp.

11. Gharbi, A. 2012. Evaluation of durum wheat genotype for drought and salinity stress in lab and greenhouse conditions. Thesis of M. of Sc. In plant breeding, Islamic Azad University, Tabriz Branch, 97 pp (In Persian).

12. Hashemzadeh, J. and H. Monirifar. 2016. Agro-morphological traits variation in some lentil landrace cultivars from northwest of Iran. Journal of Crop Breeding, 8: 102-111 (In Persian).

13. Kafi, M., H. Hosseini, A. Masomi and A. Nezami. 2006. Physiological effects of drought stress induced of PEG on lentil genotypes germination. Journal of Iran Agronomic Researches, 3: 69-80.

14. Kagan, K., T. Karakoy, A. Bakoglu 3 and M. Akçura. 2010. Determination of salinity tolerance of some lentil (Lens culinaris M.) varieties. Journal of Food Agriculture \& Envronment, 8: 140-14.

15. Mahajan, S. and N. Tutejan. 2005. Cold, salinity and drought stresses: An overview. Archives of Biochemistry and Biophysics, 444: 139-158.

16. Manchanda, G. and N. Garg. 2008. Salinity and its effects on the functional biology of legumes. Acta Physiologiae Plantarum, 30: 595- 618.

17. Okcu, G., M.D. Kaya and M. Atak. 2005. Effect of salt and drought stress on germination and seedling growth of pea (Pisum aestivum L.) Turkish journal of agriculture and forestry, 29: 237- 241.

18. Puppala, N., J.L. Poindexter and H.L. Bhardwaj. 1999. Evaluation of salinity tolerance of canola germination. In: J. Janick (ed.) Perspectives on new crops and new uses. ASHS Press, Alexardria, 251-253.

19. 19 Rehman, S., P.J.C. Harris, W.F. Bourne and J. Wikin. 1996. The effect of sodium chloride on germination and the potassium and calcium contents of Acacia seeds. Seed Science \& Technology, 25: 4557.

20. Salehi, M., F. Shekari and A. Hagnazari. 2008. Study of drought tolerance by use stability test of cell membrane and germination index in lentil genotypes. Journal of Agricultural Sciences and Natural Resources, 14: 39-50 (In Persian).

21. Sarker, A., A. Ayogan, S.H. Sabaghpour, I. Kusmnoglu, B. Sakr, W. Erskine and J. Muehlbauer. 2004. Lentil improvement for the benefit of highland farmers. In Proceedings of the $4^{\text {th }}$ International Crop Science Congress. Brisbane, Australia, 271-278.

22. Soltani, A., F.R. Koie, K. Ghassemi and M. Moghaddam. 2001. A simulation study of chickpea crop response to limited irrigation in semi-arid environments. Agricultural Water Management, 49: 225-237.

23. Takel, A. 2000. Seedling emergence and growth of sorghum genotypes under variable soil moisture deficit. Agronomy Journal, 48: 95-102.

24. Tesfaye, A., Y. Petros and H. Zeleke. 2014. Screening some accessions of lentil (Lens Culinaris M.) for salt tolerance at germination and early seedling stage In Eastern Ethiopia. International Journal of Technology Enhancements and Emerging Engineering Research, 2: 106-113.

25. Turk, M.A., A.R.M. Tahawa and K.D. Lee. 2004. Seed germination and seedling growth of three lentil cultivars under moisture stress. Asian Journal of Plant Sciences, 3: 394-397.

26. Ul-Haq, A., R. Vamil and R. Agnihotri. 2010. Effect of osmotic stress (PEG) on germination and seedling survival of lentil (Lens culinaris M.). Research Journal of Agricultural Sciences, 1:201-204.

27. Valadyani, A., A. Hasanzadeh and M. Tajbakhsh. 2006. Study of salinity stress effects on germination and seedling growths of new hi yields variety of canola. Journal of Researches and Construction (Agronomy and horticulture), 66: 23-31.

28. Valizadeh, M. and M. Moghaddam. 2009. Experimental designs in agriculture ( $4^{\text {th }}$ Edition). Tabriz, Parivar press, $451 \mathrm{pp}$ (In Persian).

29. Vanda, M., M. Khodambashi, S. Houshmand, B. Shiran and R. Amiri Fahliani. 2016. Relationship between grain yield and its components in two F3 lentil (Lens Culinaris Medik) populations. Journal of Crop Breeding, 8: 140-148 (In Persian).

30. Yagmur, M. and D. Kaydan. 2008. Early seedling growh and relative water content of-Triticale varieties under osmotic stress of water and $\mathrm{NaCl}$. Research Journal of Agriculture and Biological Sciences, 4: 767772. 


\title{
Germination Response and Seedling Traits of Lentil Landrace Genotypes to Drought and Salinity Stress and Path Analysis of Seedling Weight under Laboratory Conditions
}

\section{Varahram Rashidi}

\author{
Associate professor, of Department of Agronomy and Plant Breeding, Tabriz Brach, Islamic Azad University, \\ Tabriz, Iran (Corresponding Author: Rashidi.varahram@gmail.com) \\ Receive: January 18, 2016 \\ Accepted: February 26, 2017
}

\begin{abstract}
In order to evaluate of lentil landrace genotypes response via germination and seedling traits to drought and salinity stress, one factorial experiment with three factors (drought, salinity and genotypes) on bias Completely Randomized Design was conducted with three replications at the laboratory of agricultural faculty of Islamic Azad University of Tabriz branch. The levels of factors were including two level of drought stress (control and -8 bar by $\mathrm{PEG}_{6000}$ ), three level of salinity $(0,2$ and $4 \mathrm{ds} / \mathrm{m}$ by water of Oromyieh lake) and 15 landraces genotypes. Variance analysis result showed genotypexdrought $\times$ salinity interaction was significant for all studied traits, which shows the different responses of genotypes to drought and salinity stress for all traits. Mean comparison of traits for interaction of genotype $\times$ drought $\times$ salinity showed that germination and seedling traits value in all genotypes was significantly reduced; however, this reduction was different in different genotypes. So Kaleybar and Shavi Varzeghan genotype expressed least reduction of these traits. The regression and path analysis for seedling weight showed length of root had the highest direct on seedling weight under drought and salinity stress.
\end{abstract}

Keywords: Drought, Lentil, Path analysis, Regression, Salinity 